CHRONIC OBSTRUCTIVE PULMONARY DISEASE

\title{
Abdominal muscle and quadriceps strength in chronic obstructive pulmonary disease
}

\author{
W D-C Man, N S Hopkinson, F Harraf, D Nikoletou, M I Polkey, J Moxham
}

Thorax 2005;60:718-722. doi: 10.1136/thx.2005.040709

\begin{abstract}
See end of article for authors' affiliations

Correspondence to: Dr W D-C Man, Respiratory Muscle Laboratory, Guy's, King's and St Thomas' School of Medicine, King's College Hospital, Bessemer Road, London SE5 9PJ, UK; william.man@kcl.ac.uk

Received 23 January 2005 Accepted 19 May 2005

Published Online First 27 May 2005
\end{abstract}

\begin{abstract}
Background: Quadriceps muscle weakness is common in chronic obstructive pulmonary disease (COPD) but is not observed in a small hand muscle (adductor pollicis). Although this could be explained by reduced activity in the quadriceps, the observation could also be explained by anatomical location of the muscle or fibre type composition. However, the abdominal muscles are of a similar anatomical and fibre type distribution to the quadriceps, although they remain active in COPD. Cough gastric pressure is a recently described technique that assesses abdominal muscle (and hence expiratory muscle) strength more accurately than traditional techniques. A study was undertaken to test the hypothesis that more severe weakness exists in the quadriceps than in the abdominal muscles of patients with COPD compared with healthy elderly controls.

Methods: Maximum cough gastric pressure and quadriceps isometric strength were measured in 43 patients with stable COPD and 25 healthy elderly volunteers matched for anthropometric variables.

Results: Despite a significant reduction in mean quadriceps strength $(29.9 \mathrm{~kg} v 41.2 \mathrm{~kg} ; 95 \% \mathrm{Cl}-17.9$ to $-4.6 ; p=0.001)$, cough gastric pressure was preserved in patients with COPD $\left(227.3 \mathrm{~cm} \mathrm{H}_{2} \mathrm{O} v\right.$ $204.8 \mathrm{~cm} \mathrm{H}_{2} \mathrm{O} ; 95 \% \mathrm{Cl}-5.4$ to $50.6 ; p=0.11$ ).

Conclusions: Abdominal muscle strength is preserved in stable COPD outpatients in the presence of quadriceps weakness. This suggests that anatomical location and fibre type cannot explain quadriceps weakness in COPD. By inference, we conclude that disuse and consequent deconditioning are important factors in the development of quadriceps muscle weakness in COPD patients, or that activity protects the abdominal muscles from possible systemic myopathic processes.
\end{abstract}

A Ithough skeletal muscle dysfunction is well recognised in chronic obstructive pulmonary disease (COPD), most research has concentrated on the quadriceps because it is a primary locomotor muscle and readily accessible. Controversy remains as to whether the mechanisms of skeletal muscle dysfunction are local, systemic, or both. A predominantly systemic process would result in a widely distributed loss of muscle function, whereas chronic inactivity as a result of breathlessness and subsequent deconditioning would result in dysfunction predominantly in the locomotor muscles such as the quadriceps. We have previously shown that, in stable non-weight losing patients with COPD, adductor pollicis strength is preserved despite significant weakness in the quadriceps. ${ }^{2}$ Although this would suggest that inactivity (and subsequent disuse atrophy) is the dominant aetiology, it is possible that a systemic process may have a predilection for certain muscle fibre types. Whereas the adductor pollicis is composed principally of type I fibres, the quadriceps consists of $43 \%$ type I fibres and 57\% type II fibres. ${ }^{3}$ Similarly, the adductor pollicis is located distally while the quadriceps is a proximal muscle. Some myopathic processes such as steroid myopathy are known to preferentially affect proximal musculature. ${ }^{4}$

The expiratory muscles, of which the most important are those of the anterior abdominal wall, are proximally located and have a similar muscle fibre type distribution to the quadriceps. ${ }^{3}$ However, whereas the quadriceps muscle is chronically underused, the abdominal muscles contract during coughing (a frequent symptom in COPD) and are recruited during exercise, ${ }^{5}$ and may often be active at rest. ${ }^{6}$ Hence, evidence of abdominal muscle weakness in conjunction with quadriceps weakness would support the hypothesis that systemic factors are important in causing weakness, whereas preserved abdominal muscle strength would by inference support the notion that reduced use is a necessary condition for the development of quadriceps weakness.

Several previous studies have measured expiratory muscle strength in COPD, ${ }^{7-17}$ but there is no consensus over whether weakness is present, and few have simultaneously measured quadriceps strength. The differences between studies may reflect patient selection and the matching of appropriate control groups. The choice of test to assess expiratory muscle strength and familiarity of subjects with the test may also be relevant.

In past studies maximum expiratory mouth pressure (PEmax) has been used to assess expiratory muscle strength. This well established, non-invasive, and reproducible manoeuvre excludes expiratory muscle weakness when normal values are obtained, but care is needed when interpreting low values. In the evaluation of expiratory muscle weakness, PEmax has a positive predictive value of only $58 \%{ }^{18} \mathrm{~A}$ recently introduced test involving measurement of cough gastric pressure is a more natural manoeuvre which is easier for patients to perform and has a higher positive predictive value (94\%) in detecting abdominal (and hence expiratory) muscle weakness. ${ }^{18}$

To clarify whether expiratory muscle strength is preserved or reduced in the presence of quadriceps weakness, we measured cough gastric pressure and quadriceps maximum voluntary contraction force in a group of COPD patients and age matched healthy controls. Data obtained from some of the healthy elderly controls contributed to the normal values

\footnotetext{
Abbreviations: BMI, body mass index; COPD, chronic obstructive pulmonary disease; cough Pga, cough gastric pressure; $\mathrm{FEV}_{1}$, forced expiratory volume in 1 second; FFM, fat free mass; FFMl, fat free mass index; PEmax, maximum expiratory mouth pressure; QMVC, quadriceps maximum voluntary contraction; TLC, total lung capacity
} 
of cough gastric pressure reported in a previous study, ${ }^{18}$ and data obtained from some of the COPD patients contributed to baseline measurements reported in previous studies..$^{19} 20$

\section{METHODS}

Forty three stable patients with COPD referred to a pulmonary rehabilitation programme, with no infective exacerbation in the preceding 4 weeks, were recruited to the study. All had a smoking history of at least 20 pack-years with a clinical course consistent with the British Thoracic Society criteria for diagnosis, ${ }^{21}$ and complained of significant functional limitation due to dyspnoea. Twenty five healthy active elderly volunteers with no history of respiratory disease were also recruited. Study participants were free from comorbidities that could limit mobility or reduce muscle strength. The local research ethics committee of King's College Hospital approved the study and all participants gave informed consent.

Expiratory muscle strength was assessed in each participant by recording the cough gastric pressure (cough Pga). Quadriceps muscle strength was measured by recording the isometric quadriceps maximum voluntary contraction of the dominant leg. Fat free mass (FFM) was estimated using bioelectrical impedance analysis and disease and age specific regression equations. ${ }^{22}{ }^{23}$ FFM was normalised to height by calculating the fat free mass index (FFMI = FFM $(\mathrm{kg}) /$ height $\left.(\mathrm{m})^{2}\right)$. Spirometric parameters and lung volumes obtained from body plethysmography were measured in the COPD patients.

Cough Pga was measured as previously described ${ }^{18}$ using a balloon catheter passed nasally following local anaesthesia of the nasal mucosa and pharynx. The tip of the balloon was placed $70 \mathrm{~cm}$ from the nostril and correct placement in the stomach was confirmed by a positive deflection during a sniff manoeuvre. Seated subjects were asked to perform maximal single coughs at 30 second intervals until no further increase in cough Pga was observed. The investigator gave verbal encouragement throughout the study and cough Pga manoeuvres were displayed on a computer screen to provide visual feedback for the subject.

Isometric quadriceps maximum voluntary contraction (QMVC) was studied using the technique previously described by Polkey and colleagues ${ }^{24}$ with a specially designed chair from which the back was removed and laid flat. All subjects were studied supine or semi-supine (based on subject comfort), keeping the knee flexed at $90^{\circ}$ over the end of the chair. An inextensible strap was placed around the ankle and connected to a strain gauge, mounted to the back of the chair so that the strap ran perpendicular to the ankle and gauge. A restraining belt was used to fix the pelvis and lower trunk to minimise unwanted movement. Subjects were asked to extend their knee maximally against the strap.
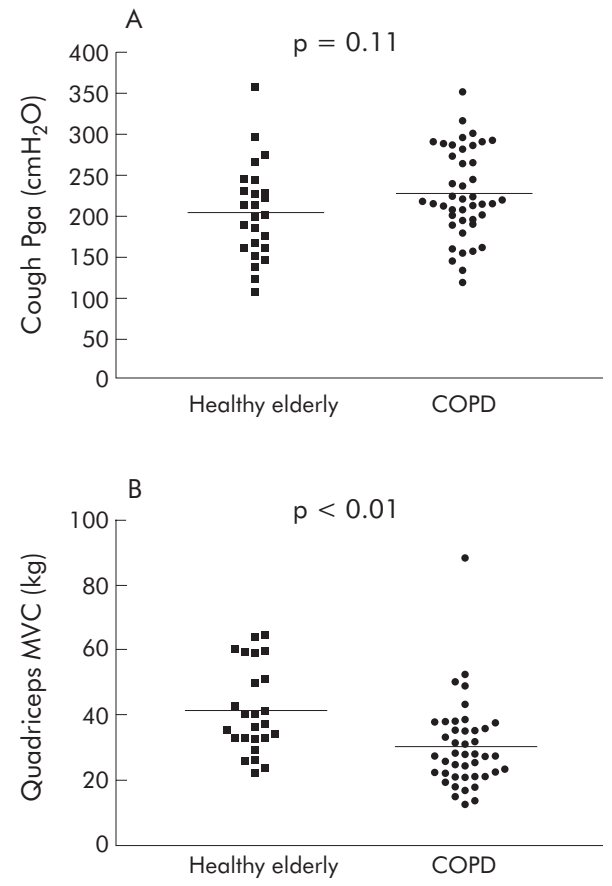

Figure 1 Scatterplots showing individual data and group means of expiratory muscle and quadriceps strength in healthy elderly controls and patients with chronic obstructive pulmonary disease (COPD). Cough $\mathrm{Pga}$, cough gastric pressure; quadriceps $M V C$, isometric quadriceps maximum voluntary contraction.

Consecutive efforts were made at 30 second intervals, with visual feedback and verbal encouragement from the investigator, until no further increase in QMVC occurred.

\section{Data analysis}

Statistical analysis was performed using SPSS Version 11.5 for Windows. As data were normally distributed, differences between the COPD and control groups were compared using unpaired $t$ tests.

\section{RESULTS}

Anthropometric data for the COPD patients $(n=43)$ and healthy elderly control subjects $(n=25)$ are summarised in table 1. The two groups were well matched for sex, height, weight, BMI, FFM, and FFMI. Twenty five of the COPD patients had received oral prednisolone in the preceding year with a mean daily dose of $3.26 \mathrm{mg}$ (four were on maintenance oral corticosteroids and 21 had received corticosteroid burst regimens for an infective exacerbation).

Table 1 Anthropometric and lung function measurements in study participants

\begin{tabular}{lllll}
\hline & $\begin{array}{l}\text { Healthy elderly } \\
\text { control group }\end{array}$ & COPD group & $\begin{array}{l}\text { Mean difference } \\
(95 \% \mathrm{CI})\end{array}$ & p value \\
\hline Sex $(\mathrm{M} / \mathrm{F})$ & $16 / 9$ & $30 / 13$ & - & $0.62^{*}$ \\
Age (years) & $67.6(14.1)$ & $64.6(10.1)$ & $-3.0(-8.9$ to 2.9$)$ & 0.31 \\
Height $(\mathrm{m})$ & $1.69(0.10)$ & $1.68(0.08)$ & $-0.01(-0.06$ to 0.03$)$ & 0.58 \\
Weight $(\mathrm{kg})$ & $74.3(13.1)$ & $69.0(16.1)$ & $-5.3(-12.9$ to 2.2$)$ & 0.16 \\
BMI $\left(\mathrm{kg} / \mathrm{m}^{2}\right)$ & $25.8(3.0)$ & $24.5(5.8)$ & $-1.3(-3.8$ to 1.2$)$ & 0.29 \\
FFM $(\mathrm{kg})$ & $50.3(12.6)$ & $46.7(7.0)$ & $-3.6(-8.4$ to 1.1$)$ & 0.13 \\
FFMI $\left(\mathrm{kg} / \mathrm{m}^{2}\right)$ & $17.3(2.6)$ & $16.5(2.2)$ & $-0.8(-1.9$ to 0.4$)$ & 0.20 \\
FEV $(\%$ predicted) & - & $35.4(18.2)$ & - & - \\
TLC (\% predicted) & - & $125(20)$ & - & - \\
\hline
\end{tabular}

Data expressed as mean (SD) or mean difference (95\% confidence interval).

BMI, body mass index; FFM, fat free mass; FFMI, fat free mass index; FEV 1 , forced expiratory volume in 1 second; TLC, total lung capacity.

Unpaired $t$ tests used throughout except ${ }^{*} \chi^{2}$ test. 
Seven patients were receiving domiciliary long term oxygen therapy. Nutritional depletion was considered to be present if FFMI was $<15$ for women or $<16$ for men. ${ }^{22}$ Based on these criteria, 15 of the 43 patients were nutritionally depleted.

Figure 1 shows individual data and group means for cough Pga and QMVC. Mean (SD) QMVC was reduced in patients with COPD (29.9 (13.2) kg) compared with the control group (41.2 (13.3) kg; 95\% CI -17.9 to $-4.6 ; p=0.001)$. When stratified for sex, QMVC remained significantly reduced in both male and female COPD patients compared with their healthy counterparts (female: 23.2 (10.2) kg $v 29.6$ (6.0) kg, $\mathrm{p}=0.03$; male: 32.8 (13.4) kg $v 47.7$ (11.8) kg, $\mathrm{p}=0.0006$ ). There was a trend for cough Pga to be increased in the COPD group, although this did not reach statistical significance (227.3 (54.2) $\mathrm{cm} \mathrm{H}_{2} \mathrm{O} v 204.8$ (58.3) $\mathrm{cm} \mathrm{H}_{2} \mathrm{O} ; 95 \% \mathrm{CI}-5.4$ to $50.6 ; \mathrm{p}=0.11)$. This was not affected by stratification for sex (female: 197.5 (50.4) kg $v 167.0$ (44.3) kg, $\mathrm{p}=0.16$; male: $240.3(51.2) \mathrm{kg} v 226.0(55.4) \mathrm{kg}, \mathrm{p}=0.39)$.

There was no linear relationship between FFMI and either QMVC $\left(r^{2}<0.01 ; \mathrm{p}=0.53\right)$ or cough Pga $\left(r^{2}=0.02 ; \mathrm{p}=0.41\right)$ in the COPD patients. No significant relationship was found between percentage predicted total lung capacity (TLC) and cough Pga $\left(r^{2}<0.001 ; \mathrm{p}=0.85\right)$.

\section{DISCUSSION}

The principal finding of this study is that cough Pga is normal in COPD patients compared with healthy elderly controls matched for age and FFM, despite a substantial reduction in quadriceps strength. These data support the hypothesis that a systemic process alone is not responsible for quadriceps weakness in non-weight losing patients with COPD. Before enlarging on these points, it is appropriate to discuss the methodology.

\section{Cough Pga as an index of expiratory muscle strength} In this study cough Pga was used as an index of expiratory muscle strength rather than the more traditional maximum expiratory mouth pressure (PEmax). PEmax is the only commonly used test for assessment of expiratory muscle strength and has many advantages. It is non-invasive, reproducible, and normal values are well described. When high values of PEmax are obtained, expiratory muscle weakness can be excluded..$^{18}$ However, care is required in interpreting low values as many subjects may underperform this manoeuvre. In a recent study of 293 patients referred for respiratory muscle testing, over $40 \%$ of patients with a low PEmax had a normal cough Pga, ${ }^{18}$ demonstrating that PEmax commonly underestimates true expiratory muscle strength. Measurement of cough Pga offers an alternative to PEmax as the principal expiratory muscles-the abdominal musclesare strongly recruited in cough. While it is not a nonvolitional technique, it has the advantage that, as a natural dynamic manoeuvre, cough is technically easier to perform than PEmax while retaining similar between occasion reproducibility. ${ }^{18}$ The obvious disadvantage of the technique is that it is invasive, requiring the placement of a balloon catheter. However, in this clinical research study all participants tolerated the procedure well and there were no adverse events. The expiratory muscles, like the quadriceps, can be assessed non-volitionally by magnetic stimulation of the lower thoracic nerve roots. ${ }^{25}$ However, this technique, unlike stimulation of the femoral nerve or phrenic nerves, is not supramaximal and hence is less useful when precisely quantifying expiratory muscle strength.

A possible confounding factor is increased lung volume and the effect on the length-tension relationship of the abdominal muscles. Cough Pga measurements were performed at or near TLC, and one would expect the patients with COPD, with their increased TLC, to produce higher gastric pressures during the manoeuvres. However, previous studies from our laboratory have shown that lung volume exerts only a relatively modest effect on Pga. ${ }^{26}{ }^{27}$ Using paired magnetic stimuli of the thoracic nerve roots, Pga only increases about $17 \%$ from functional residual capacity to TLC. ${ }^{26}$ In this study no significant relationship was found between TLC and cough Pga.

\section{Patient selection}

The patients recruited for this study were sedentary, clinically stable patients with moderate to severe COPD, as commonly seen in respiratory outpatient clinics. Mean forced expiratory volume in 1 second $\left(\mathrm{FEV}_{1}\right)$ was $35 \%$ predicted (range $12-$ $58 \%$ ), and all had been referred to a pulmonary rehabilitation programme. Although the BMI, FFM, and FFMI of the patients were not significantly lower than the control group, $35 \%$ of the patients met standard criteria for nutritional depletion, supporting previous observations of body composition in patients with stable COPD. ${ }^{28}$ A limitation of our study is that we did not make formal objective measurements of daily activity levels. However, we believe it is reasonable to assume our patients were more sedentary than their healthy counterparts. $^{29}$ All patients complained of exertional dyspnoea and reduced exercise tolerance (hence their referral to pulmonary rehabilitation), while all the healthy elderly controls were either in part or full time employment or led active social lives.

\section{Previous studies of expiratory muscle strength in COPD}

Several previous studies have measured expiratory muscle strength in $\mathrm{COPD}^{7-17}$ but no consensus has been reached over whether weakness is present. Some investigators have described significant expiratory muscle weakness, ${ }^{8-10}{ }^{13-16}$ whereas others have shown normal strength ${ }^{11}{ }^{17}$ or, indeed, increased strength. ${ }^{7}$ These differences may be due to patient selection, the absence of appropriate healthy control groups, or differences in nutritional status. An alternative explanation may in part be the choice of PEmax as the test of expiratory muscle strength, and this study is novel in that it is the first to measure cough Pga in both COPD patients and healthy controls. Only one other study has used the cough manoeuvre to measure expiratory muscle strength in COPD. In 1968 Byrd and Hyatt measured cough oesophageal pressures in 31 patients with chronic obstructive lung disease $^{7}$ and showed, as in our study, higher cough pressures than corresponding mouth static pressures, although no measurements in controls were made nor measurements of quadriceps strength in the patients.

Few studies have directly compared the expiratory muscles with the quadriceps muscle in patients with COPD. Gosselink and colleagues measured respiratory muscle, handgrip, and quadriceps strength as well as exercise capacity in 41 patients with COPD and found similar reductions in PEmax and isometric quadriceps force. ${ }^{9}$ However, FFM measurements were not made and the muscle strength data were compared with historical normal values. The same group later quantified respiratory and peripheral muscle strength in 40 COPD patients and 22 healthy elderly controls. ${ }^{10}$ Although they concluded that muscle weakness did not affect all muscles to a similar extent, the reduction in strength in the expiratory muscles was similar to the reduction seen in knee extension. However, their patients were not matched to the controls in terms of weight or BMI, hence the influence of nutritional status and FFM as confounding factors on expiratory muscle strength could not be excluded. In contrast, the patients in the current study had normal cough Pga values but significantly reduced quadriceps maximum 
voluntary contraction force compared with controls matched for FFM.

\section{Significance of findings}

In the present study it was observed that cough Pga in the COPD group was preserved compared with pressures obtained from a group of healthy active elderly subjects matched for age, sex, and anthropometric values, despite a significant reduction in quadriceps strength. This supports the hypothesis that local rather than systemic factors have a principal role in initiating muscle dysfunction. An obvious local factor is inactivity and subsequent muscle deconditioning due to the sedentary lifestyles of patients locked in a downward spiral of breathlessness. Dysfunction would be expected to be greatest in the muscles that are least used-for example, the locomotor muscles such as the quadriceps. Unlike the quadriceps muscle, the abdominal muscles are not chronically underused and are frequently recruited during rest and exercise. ${ }^{5}{ }^{6}$

This hypothesis is supported by previous studies showing the unequal distribution of muscle weakness in COPD-in particular, the strength of the upper extremity muscles being relatively preserved compared with those of the lower extremity. ${ }^{10}$ Using the non-volitional technique of supramaximal magnetic nerve stimulation to exclude the confounding effects of patient motivation, we have previously shown that both twitch adductor pollicis tension and twitch transdiaphragmatic pressures are normal in patients with COPD compared with healthy elderly controls, despite a 30\% reduction in twitch quadriceps force. ${ }^{2}$ One potential criticism of that study is that the adductor pollicis and quadriceps muscles vary in their fibre type distribution: the adductor pollicis is composed principally of type I fibres (80\%) whereas the quadriceps consists of $43 \%$ type I fibres and $57 \%$ type II fibres. ${ }^{3}$ The present study largely addresses this criticism as the abdominal muscles have a similar fibre type distribution to the quadriceps, comprising $46 \%$ type I fibres and 54\% type II fibres. ${ }^{3}$

Although it has been postulated that inactivity may play a major factor in skeletal muscle dysfunction in COPD patients, there is some evidence that this may not explain all observed abnormalities. Maltais and colleagues have shown modifications in the myosin heavy chain profile in the vastus lateralis of patients with COPD compared with healthy subjects despite only modest differences in physical activity. ${ }^{30}$ Studies on emphysematous hamsters have shown reduced oxidative capacity of their hindlimb muscles despite the absence of a reduction in their level of activity compared with control animals. ${ }^{31}$ Other local factors such as local exercise induced oxidative stress may also be involved in initiating muscle dysfunction. ${ }^{32}$

Controversy remains over whether a systemic inflammatory response is a major aetiological factor in the skeletal muscle dysfunction commonly seen in COPD. Indirect evidence supporting this hypothesis comes from the observation that levels of tumour necrosis factor $\alpha$ are raised in patients who fail to gain weight during a rehabilitation and refeeding programme, ${ }^{33}$ while interleukin 8 levels are inversely related to quadriceps strength in patients admitted with an exacerbation of COPD. ${ }^{34}$ Increased levels of interleukin 6 are also associated with radiological evidence of quadriceps wasting in $\mathrm{COPD}^{35}$ and with reduced lean body mass. ${ }^{36}$ Levels of inflammatory cytokines were not measured in this study, and hence a systemic inflammatory process cannot be excluded. However, the results from this study suggest that activity protects the muscles against any putative systemic process. Even in situations of increased systemic inflammation such as soon after the onset of an acute exacerbation, increased activity in the form of a pulmonary rehabilitation programme can lead to highly significant improvements in exercise capacity and health status. ${ }^{37}$ Available data suggest that any future therapeutic interventions targeted at the inflammatory process will need to be accompanied by exercise training.

In summary, cough gastric pressures are normal in patients with stable COPD despite the presence of significant weakness of the quadriceps. This observation supports the hypothesis that disuse is necessary for the development of skeletal muscle weakness in COPD patients.

\section{Authors' affiliations}

W D-C Man, N S Hopkinson, F Harraf, D Nikoletou, M I Polkey, J Moxham, Respiratory Muscle Laboratory, Guy's, King's and St Thomas' School of Medicine, King's College Hospital and Royal Brompton Hospital, London, UK

WD-CM is a Clinical Research Training Fellow of the Medical Research Council (UK). NSH is funded by the Wellcome Trust and the ENIGMA program (QLK6-CT-2002-02285) of the European Union.

Competing interests: the authors have no competing interests to declare.

\section{REFERENCES}

1 Bernard S, LeBlanc $P$, Whittom $F$, et al. Peripheral muscle weakness in patients with chronic obstructive pulmonary disease. Am J Respir Crit Care Med 1998; 158:629-34.

2 Man WD, Soliman MG, Nikoletou D, et al. Non-volitional assessment of skeletal muscle strength in patients with chronic obstructive pulmonary disease. Thorax 2003;58:665-9.

3 Johnson MA, Polgar J, Weightman D, et al. Data on the distribution of fibre types in thirty-six human muscles. An autopsy study. J Neurol Sci 1973;18:111-29.

4 Dekhuijzen PN, Decramer M. Steroid-induced myopathy and its significance to respiratory disease: a known disease rediscovered. Eur Respir $J$ 1992:5:997-1003.

5 Man WD, Mustfa N, Nikoletou D, et al. Effect of salmeterol on respiratory muscle activity during exercise in poorly reversible COPD. Thorax 2004;59:471-6.

6 Ninane V, Rypens F, Yernault JC, et al. Abdominal muscle use during breathing in patients with chronic airflow obstruction. Am Rev Respir Dis 1992; 146:16-21.

7 Byrd RB, Hyatt RE. Maximal respiratory pressures in chronic obstructive lung disease. Am Rev Respir Dis 1968;98:848-56.

8 Ferrari K, Goti P, Misuri G, et al. Chronic exertional dyspnea and respiratory muscle function in patients with chronic obstructive pulmonary disease. Lung 1997; 175:311-9.

9 Gosselink R, Troosters T, Decramer M. Peripheral muscle weakness contributes to exercise limitation in COPD. Am J Respir Crit Care Med 1996; 153:976-80.

10 Gosselink R, Troosters T, Decramer M. Distribution of muscle weakness in patients with stable chronic obstructive pulmonary disease. J Cardiopulm Rehabil 2000;20:353-60.

11 Heijdra YF, Pinto-Plata V, Frants R, et al. Muscle strength and exercise kinetics in COPD patients with a normal fat-free mass index are comparable to control subjects. Chest 2003;124:75-82.

12 Piitulainen E, Areberg J, Linden M, et al. Nutritional status and muscle strength in patients with emphysema and severe alpha(1)-antitrypsin deficiency. Chest 2002;122:1240-6

13 Ramirez-Sarmiento A, Orozco-Levi M, Barreiro E, et al. Expiratory muscle endurance in chronic obstructive pulmonary disease. Thorax 2002;57:132-6.

14 Rochester DF, Braun NM, Arora NS. Respiratory muscle strength in chronic obstructive pulmonary disease. Am Rev Respir Dis 1979;119:151-4.

15 Rochester DF, Braun NM. Determinants of maximal inspiratory pressure in chronic obstructive pulmonary disease. Am Rev Respir Dis 1985;132:42-7.

16 Weiner $\mathbf{P}$, Magadle R, Beckerman $M$, et al. Specific expiratory muscle training in COPD. Chest 2003;124:468-73.

17 Nishimura Y, Tsutsumi M, Nakata H, et al. Relationship between respiratory muscle strength and lean body mass in men with COPD. Chest 1995; 107:1232-6.

18 Man WD, Kyroussis D, Fleming TA, et al. Cough gastric pressure and maximum expiratory mouth pressure in humans. Am J Respir Crit Care Med 2003; 168:714-7

19 Hopkinson NS, Man WD, Dayer MJ, et al. Acute effect of oral steroids on muscle function in chronic obstructive pulmonary disease. Eur Respir J 2004;24:137-42.

20 Hopkinson NS, Nickol AH, Payne J, et al. Angiotensin converting enzyme genotype and strength in chronic obstructive pulmonary disease. Am J Respir Crit Care Med 2004; 170:395-9.

21 COPD Guidelines Group of the Standards of Care Committee of the BTS. BTS guidelines for the management of chronic obstructive pulmonary disease. Thorax 1997;52(Suppl 5):S1-28. 
22 Steiner MC, Barton RL, Singh SJ, et al. Bedside methods versus dual energy Xray absorptiometry for body composition measurement in COPD. Eur Respir $J$ 2002; 19:626-31.

23 Deurenberg $\mathbf{P}$, van der Kooij K, Evers $P$, et al. Assessment of body composition by bioelectrical impedance in a population aged greater than 60 y. Am J Clin Nutr 1990;51:3-6.

24 Polkey MI, Kyroussis D, Hamnegard CH, et al. Quadriceps strength and fatigue assessed by magnetic stimulation of the femoral nerve in man. Muscle Nerve 1996;19:549-55.

25 Man WD, Moxham J, Polkey MI. Magnetic stimulation for the measurement of respiratory and skeletal muscle function. Eur Respir J 2004;24:846-60.

26 Kyroussis D, Polkey MI, Mills GH, et al. Simulation of cough in man by magnetic stimulation of the thoracic nerve roots. Am J Respir Crit Care Med 1997; 156:1696-9.

27 Polkey MI, Luo Y, Guleria R, et al. Functional magnetic stimulation of the abdominal muscles in humans. Am J Respir Crit Care Med 1999:160:513-22.

28 Schols AM, Soeters PB, Dingemans AM, et al. Prevalence and characteristics of nutritional depletion in patients with stable COPD eligible for pulmonary rehabilitation. Am Rev Respir Dis 1993;147:1151-6.

29 Schonhofer B, Ardes P, Geibel M, et al. Evaluation of a movement detector to measure daily activity in patients with chronic lung disease. Eur Respir $J$ 1997; 10:2814-9
30 Maltais F, Sullivan MJ, LeBlanc $P$, et al. Altered expression of myosin heavy chain in the vastus lateralis muscle in patients with COPD. Eur Respir $J$ 1999; 13:850-4.

31 Mattson JP, Poole DC. Pulmonary emphysema decreases hamster skeletal muscle oxidative enzyme capacity. J Appl Physiol 1998:85:210-4.

32 Couillard A, Koechlin C, Cristol JP, et al. Evidence of local exercise-induced systemic oxidative stress in chronic obstructive pulmonary disease patients. Eur Respir J 2002;20:1123-9.

33 Creutzberg EC, Schols AM, Weling-Scheepers CA, et al. Characterization of nonresponse to high caloric oral nutritional therapy in depleted patients with chronic obstructive pulmonary disease. Am J Respir Crit Care Med 2000;161:745-52.

34 Spruit MA, Gosselink R, Troosters T, et al. Muscle force during an acute exacerbation in hospitalised patients with COPD and its relationship with CXCL8 and IGF-I. Thorax 2003:58:752-6.

35 Debigare R, Marquis K, Cote $\mathrm{CH}$, et al. Catabolic/anabolic balance and muscle wasting in patients with COPD. Chest 2003;124:83-9.

36 Eid AA, lonescu AA, Nixon LS, et al. Inflammatory response and body composition in chronic obstructive pulmonary disease. Am J Respir Crit Care Med 2001; 164:1414-8.

37 Man WD, Polkey MI, Donaldson N, et al. Community pulmonary rehabilitation after hospitalisation for acute exacerbations of chronic obstructive pulmonary disease: randomised controlled study. BMJ 2004;329:1209.

\section{LUNG ALERT}

\section{Comparing the Pneumonia Severity Index and CURB scores}

$\Delta$ Aujesky D, Auble TE, Yealy DM, et al. Prospective comparison of three validated prediction rules for prognosis in community-acquired pneumonia. Am J Med 2005; 1 18:384-92

A number of different models have been used to assess prognosis in pneumonia. In this study the Pneumonia Severity Index was compared with the CURB and CURB-65 severity scores in predicting 30 day mortality.

3181 patients with community acquired pneumonia were assessed between January and December 2001 in 32 emergency departments in Connecticut and Pennsylvania. Patients were assessed according to the criteria of the Pneumonia Severity Index, CURB, and CURB65 and then stratified into risk groups $\mathrm{I}-\mathrm{V}, 0-4$, and $0-5$, respectively. Statistical analysis was performed to assess 30 day mortality and the accuracy of the risk classification based on sensitivity, specificity, likelihood ratios, and predictive values. The results indicated that the Pneumonia Severity Index stratified more patients as low risk $(68 \%)$ than CURB $(51 \%)$ or CURB-65 $(61 \%)$, both $\mathrm{p}<0.001$. Low risk patients identified by the Pneumonia Severity Index had slightly lower 30 day mortality (1.4\%) than those stratified as low risk in CURB $(1.7 \%)$ or CURB-65 (1.7\%). It was found that the Pneumonia Severity Index had a higher sensitivity and lower specificity than the CURB scores. All three models had high negative predictive values $(>95 \%)$.

The absolute difference in 30 day mortality based on the three models is small and of uncertain clinical significance. However, the more complex Pneumonia Severity Index does classify many more patients as low risk and this may have beneficial cost implications in terms of outpatient treatment.

A S Patel

Senior House Officer, Royal Free Hospital, London, UK; amit.patel@royalfree.nhs.uk 